médecine/sciences $1988 ; 7: 420-426$

\title{
La tolérance des greffes de foie
}

Chez certains animaux, par exemple le porc et surtout le rat, la greffe de foie peut être tolérée spontanément dans certaines combinaisons génétiques entre les donneurs et les receveurs. Mieux, la transplantation d'un greffon hépatique peut induire chez le receveur une tolérance de transplantation spécifique des antigènes du donneur, une greffe secondaire d'un organe animal syngénique au donneur de la greffe hépatique étant alors tolérée. Les mécanismes immunologiques de ce phénomène sont encore mal compris. Chez l'homme, le phénomène de tolérance est moins spectaculaire mais explique néanmoins l'absence apparente d'influence nette sur le pronostic d'incompatibilités dans les systèmes antigéniques HLA et ABO.

\section{Didier Houssin Professeur de chirurgie diges- tive}

\section{ADRESSE ET TIRÉS A PART}

D. Houssin : clinique chirurgicale et laboracoire de recherche chirurgicale (Pr. Y. Chapuis), hôpital Cochin, 27, rue du Fau-

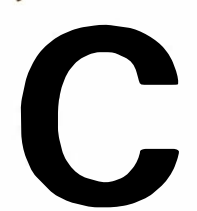

ette décennie est celle du second souffle de la transplantation d'organe. L'essor des transplantations cardia. ques et hépatiques en est la cause. Cette dernière est devenue le traitement indiscutable de nombreuses maladies hépatiques, aiguës ou chroniques, de l'adulte et de l'enfant, ainsi que de quelques maladies métaboliques d'origine hépatique. Compensation heureuse de la complexité de sa réalisation chirurgicale, la greffe de foie jouit, face aux problèmes de rejet, d'une place privilégiée comparativement aux autres organes. Cette mise au point a pour but d'éclairer les différentes formes de la tolérance des greffes hépatiques : tolérance immunologique spécifique induite après allogreffe de foie chez certains animaux, rêve de tous les transplanteurs; ou bien, plus modestement, la bonne tolérance qui, chez l'homme, facilite la réalisation de la greffe en permettant de diminuer l'intensité (et donc la toxicité) de l'immunosuppression.

Tolérance immunologique spécifique après allogreffe hépatique expérimentale

Dans plusieurs espèces animales, en l'absence de traitement immunosuppresseur, les allogreffes de foie sont rejetées de manière aiguë, comme les allogreffes d'autres organes. Cependant, en 1967, Garnier et al. [1] ont montré que, malgré l'absence de traitement immunosuppresseur, des allogreffes de foie pouvaient être tolérées de manière très prolongée chez des porcs. Cette observation a été confirmée peu de temps après par Calne et al. [2] qui ont, de plus, révélé la véritable signification de ce phénomène en démontrant que les animaux tolérant une allogreffe de foie étaient également tolérants vis-à-vis d'autres tissus provenant du même donneur et de lui seul. Le développement des techniques microchirurgicale a permis d'étudier ce phénomène chez le rat dans des conditions immunogénétiques beaucoup plus précises (figure 1).

Dans de nombreuses combinaisons donneur-receveur de rats de souche pure, mais pas dans toutes, les allogreffes de foie effectuées en l'absence de traitement immunosuppresseur ou de transfusion. sont spontanément tolérées [3, 4], alors que le rejet des allogreffes de peau ou de cœur dans les mêmes combinaisons est constant. En fait, ces allogreffes de foie présentent à partir du cinquième jour après la transplantation des signes biologiques et histologiques de rejet. A partir du $15^{\mathrm{e}}$ jour après la greffe, cependant, ceux-ci s'estompent peu à peu pour ne plus réap- 


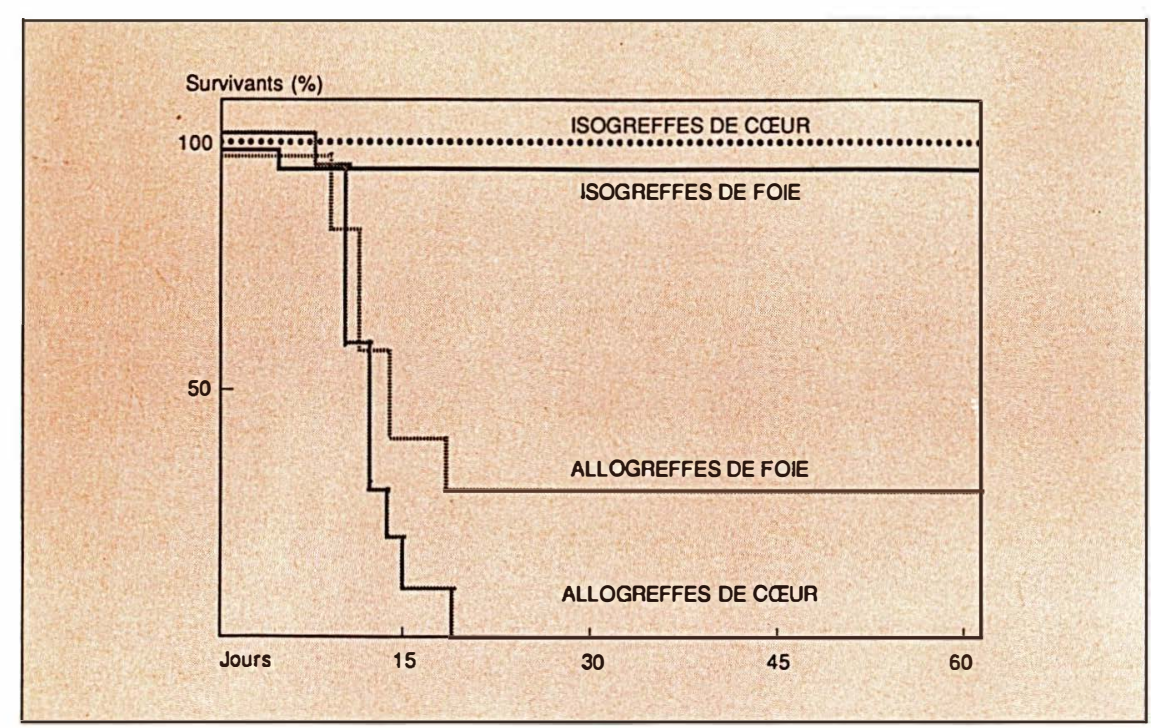

Figure 1. Dans la combinaison donneur-receveur de rats de souche pure Lewis $\rightarrow$ Brown Norway, le rejet des allogreffes de cour est constant alors qu'environ un tiers des allogreffes de foie sont tolérées en l'absence de traitement immunosuppresseur.

paraître [5]. Une analyse plus fine permet en fait d'individualiser un effet tolérigène très précoce induit par l'allogreffe de foie vis-à-vis des antigènes du donneur, puisque dans certaines combinaisons donneurreceveur une allogreffe de cœur effectuée en même temps que l'allogreffe de foie peut également être tolérée [6]. Lorsque l'allogreffe de foie est effectuée chez l'animal en train de rejeter une allogreffe de cœur préalable, il est possible d'observer dans certains cas une disparition des phénomènes de rejet au niveau du greffon cardiaque, l'allogreffe hépatique ayant alors un véritable effet immunosuppresseur [6]. Quelques semaines plus tard, l'évaluation du statut immunologique de ces animaux révèle qu'ils sont dans un état de tolérance de transplantation spécifique des antigènes du donneur: les greffes de peau ou de cœur effectuées secondairement et provenant d'un donneur génétiquement identique à celui ayant donné l'allogreffe de foie sont tolérées alors que des allogreffes de même type provenant d'un autre donneur inapparenté sont rejetées dans les délais habituels. Les lymphocytes $\mathrm{T}$ du receveur ont cependant conservé leur capacité de reconnaître les antigènes du donneur : leur prolifération est normale ou faiblement diminuée, in vitro dans une $\mathrm{m} / \mathrm{s} n^{\circ} 7$ vol. 4 , septembre 88 teurs non spécifiques qui tiennent à la trophicité du foie, et sans doute à la capacité qu'il a de régénérer au cours d'un processus de destruction hépatocytaire tel qu'un rejet aigu. Engemann et al. [9] ont ainsi montré que dans la combinaison donneurreceveur DA-Lewis (il y a des rats DA et des rats Lewis), l'allogreffe de foie n'était tolérée que si l'apport artériel au greffon était rétabli.

De nombreuses incertitudes persistent encore à ce jour, d'une part quant au mécanisme de maintien de l'état de tolérance et d'autre part quant au rôle du foie dans l'induction de cet état.

La complexité des états de tolérance immunologique est bien connue et les facteurs en cause sont probablement multiples. Plusieurs travaux ont tenté d'analyser les mécanismes responsables de la tolérance de transplantation spécifique des antigènes du donneur après allogreffe de foie chez le rat. La tolérance cellulaire peut être interprétée de deux manières: l'avortement des cellules cytotoxiques ou la présence de cellules suppressives. Certains travaux comparant les conséquences du transfert de lymphocytes obtenus à partir d'allogreffes hépatiques à celles du transfert de lymphocytes du sang périphérique, à différents moment après la greffe, suggèrent que les lymphocytes $\mathrm{T}$ cytotoxiques spécifiques des antigènes du donneur prolifèrent en effet transitoirement dans le greffon, et dans lui seul, avant d'y être détruit [10]. D'autres travaux fondés sur l'étude in vitro de cultures lymphocytaires évoquent la présence de cellules $T$ suppressives [7-9]. Quelques expériences de transfert de la tolérance par le sérum et la rapidité de l'effet immunosuppresseur observée après transplantation hépatique suggèrent qu'un mécanisme humoral pourrait être aussi impliqué. La nature de ce (ou ces) facteur(s) sérique(s) reste cependant hypothétique. L'hépatocyte murin est capable de sécréter un antigène soluble du complexe majeur d'histocompatibilité de classe I[1113]. Cependant le caractère monomorphe de cet antigène et son absence d'effet immunosuppresseur in vivo [13] ne semblent guère en faveur de son rôle dans cette tolérance. Les anticorps anti $\mathrm{CMH}$ de 
RÉFÉRENCES

1. Garnier H, Clot JP, Bertrand M, et al. Transplantation hépatique expérimentale chez le porc. CR Acad Sci 1965; 260 : 5621 . 3 .

2. Caine RY, Sells RA, Pena JR, e c al. Induction of immunological tolerance by porcine liver allografts. Nature 1969; 223 : 472-6.

3. Houssin D, Gigou M, Franco D, et al. Spontaneous long term survival of liver allograft in inbred rats. Transplant Proc 1979; $11: 567-70$.

4. Zimmermann FA, Davies HffS, Knoll PP Gokel JM, Schmidt R. Orthotopic liver allografts in the rat. The influence of strain combination on the fate of the graft. Transplantation $1984 ; 37$ : 406-10.

5. Kamada N, Davies HffS, Wight D, Culank $\mathrm{L}$, Roser B. Liver transplantation in the rat biochemical and histological evidence of complete tolerance induction in nonrejector strains. Transplantation 1983; 35: 217-21.

6. Kamada N, Wight DGD. Antigen-specific immuno-suppression induced by liver transplantation in the rat. Transplantation 1984 $38: 217-21$.

7. Houssin D, Charpentier B, Gugenheim J et al. Spontaneous long term acceptance of RT-1 incompatible liver allografts in inbred rats. Transplantation $1983 ; 36: 615-20$.

8. Kamada $\mathrm{N}$. The immunology of experimental liver transplantation in the rat. Immunology 1985 ; 55 : 369-89.

9. Engemann $R$, Ulrichs $K$, Thiede A, Muller-Ruchholtz W, Hamelmann $\mathrm{H}$. A mechanism of tolerance in arterialized rat liver transplantation. Transplant Proc 1983; 15 : 729-33.

10. Davies HffS, Kamada N, Roser BJ Mechanisms of donor-specific unresponsiveness induced by liver grafting. Transplant Proc 1983; 15 : 831-5.

11. Cosman D, Kress M, Khoury G, Jay G. Tissue-specific expression of an unusual $\mathrm{H}$. 2 (class I)-related gene. Proc Nall Acad Sci USA 1982; 79: 4947-51.

12. Spencer SC, Fabre JW. Identification in rat liver and serum of a water-soluble class I MHC molecule possibly homologous to the murine Q10 gene product. J Exp Med 1987; 165 : 1595-608.

13. Spencer SC, Fabre JW. Bulk purification of a naturally occuring soluble form of $\mathrm{RT}$. l class I major histocompatibility complex antigens from DA rat liver and studies of specific immunosuppression. Transplanta-

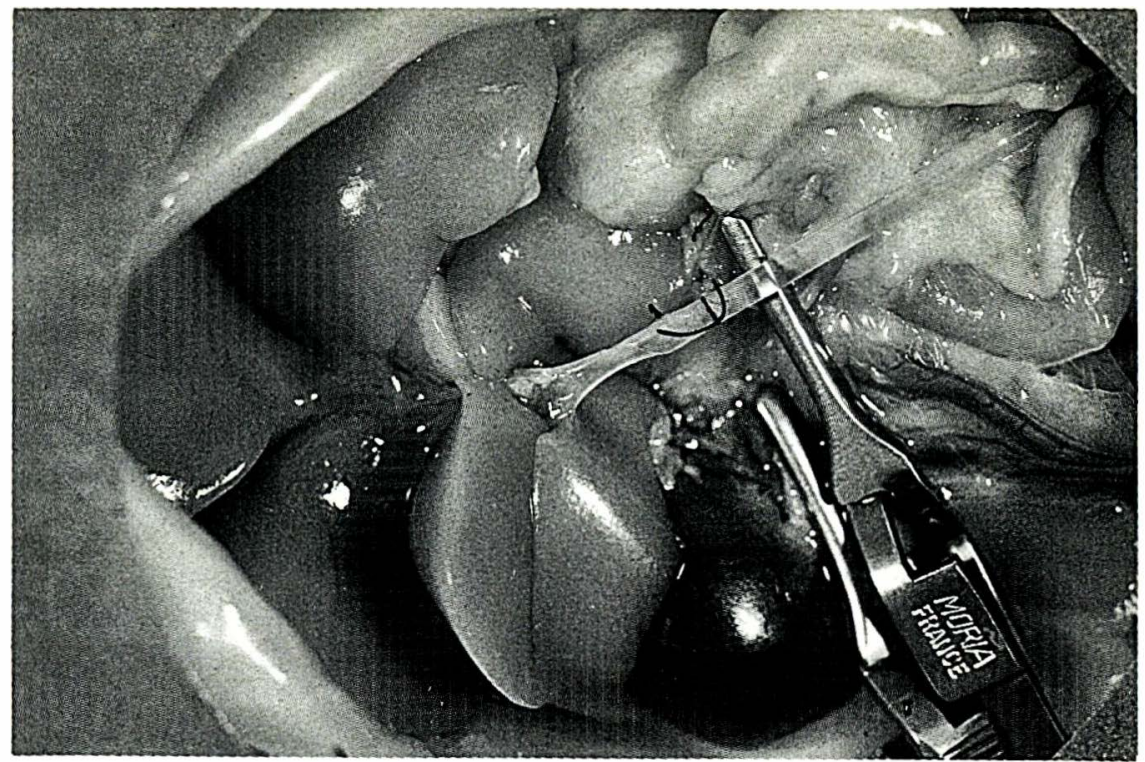

Figure 2. Transplantation hépatique orthotopique chez le rat. Lavage du greffon par la veine porte.

classe II et les complexes immuns anticorps-antigène du $\mathrm{CMH}$ de classe I ont également été incriminés sans qu'il soit possible de conclure que tel ou tel de ces facteurs joue un rôle exclusif $[8,14]$.

Les facteurs génétiques et les hypothèses immunologiques ne peuvent faire oublier le rôle essentiel du foie lui-même dans l'induction et le maintien de la tolérance de greffe. Le foie est un organe très volumineux, capable de régénérer. Ces deux caractéristiques peuvent expliquer une relative résistance au rejet, résistance probablement amplifiée par les particularités hépatiques de l'expression des antigènes d'histocompatibilité de classe II. A l'état normal chez le rat, ces antigènes sont exprimés essentiellement par les cellules dendritiques qui sont localisées aux espaces portes et absentes dans le lobule. Dans d'autres organes, comme le cœur de rat, la répartition des cellules dendritiques est beaucoup plus diffuse [15]. Dans les jours suivant une allogreffe hépatique, les antigènes de classe II apparaissent généralement au niveau des différents types cellulaires, leur expression devenant forte. Dans des allogreffes réalisées chez un donneur destiné à devenir tolérant, au contraire, l'expression des antigènes de classe II est tardive et reste très faible au niveau des hépatocytes. Il a par ailleurs été montré qu'à la fin de la deuxième semaine les cellules de Kupffer du greffon étaient remplacées par des cellules de l'hôte [16], supprimant ainsi le principal contingent cellulaire immunogène du greffon. Tous ces facteurs pourraient expliquer la relative résistance au rejet de l'allogreffe hépatique, puis la tolérance de celle-ci, fondée sur l'adaptation du greffon. Cette adaptation de l'allogreffe hépatique a été confirmée récemment par des expériences de transfert d'allogreffes hépatiques à des animaux syngéniques naïfs qui les tolèrent alors correctement [17]. En revanche, lorsqu'une allogreffe cardiaque Lewis, tolérée depuis plusieurs semaines chez un animal Brown Norway tolérant une allogreffe de foie Lewis, est transplantée chez un animal Brown Norway naïf, l'allogreffe cardiaque est rejetée dans des délais normaux [18].

\section{Tolérance des greffes hépatiques chez l'animal immunisé}

La tolérance du foie au rejet est retrouvée lorsque l'animal receveur a été préalablement sensibilisé par les antigènes du donneur. Ainsi Kamada et al. [19] ont montré que, dans la 


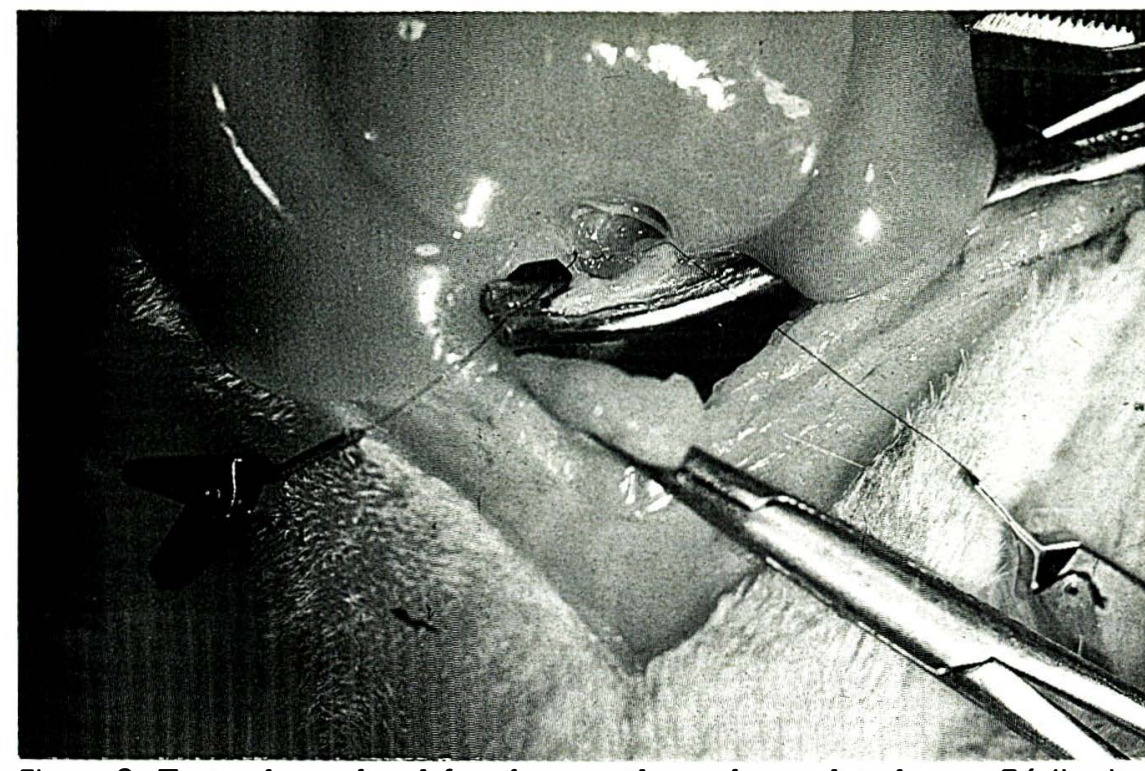

Figure 3. Transplantation hépatique orthotopique chez le rat. Réalisation de l'anastomose de la veine cave sus-hépatique.

combinaison donneur-receveur de rats $\mathrm{DA}$ et $\mathrm{PVG}^{*}$, une sensibilisation préalable du receveur PVG par une greffe de peau DA n'empêche pas la tolérance d'une greffe de foie DA réalisée quelques jours plus tard, ni l'installation d'un état de tolérance de transplantation spécifique des antigènes de DA.

Dans les situations d'hypersensibilisation allogénique[20], lorsque l'animal receveur subit par exemple plusieurs allogreffes de peau successives provenant du même donneur, une allogreffe hépatique mise en place secondairement est toujours rejetée. Cependant, contrairement à ce qui est observé pour les allogreffes cardiaques, l'allogreffe hépatique ne subit pas un rejet hyperaigu. Dans la plupart des cas, le rejet est simplement accéléré et tout se passe comme si le foie était capable de résister aux anticorps lymphocytotoxiques circulants qui sont la conséquence principale de ces états d'hypersensibilisation allogénique. L'étude du titre de ces anticorps avant et après l'allogreffe hépatique a révélé une diminution très nette au décours de l'allogreffe hépatique. Une telle baisse n'est pas observée au décours du rejet

\footnotetext{
* DA et PVG: noms de souches de rats. $\mathrm{m} / \mathrm{s} n^{\circ} 7 \mathrm{vol} .4$, seplembre 88
}

une situation particulière : si le rejet est là encore constant, il est cependant nettement moins rapide que pour le cœur [21] et il semble plus aisé d'obtenir une prolongation de la survie du greffon par des inhibiteurs des conséquences de la réaction antigène-anticorps tels que les antagonistes du facteur d'activation plaquettaire (Filipponi et al., résultats non publiés).

\section{La tolérance des greffes hépatiques chez l'homme : jusqu'à quel point?}

Le risque est grand d'imaginer transférer simplement les données tirées de l'expérimentation animale à l'activité clinique. Les données spectaculaires concernant la tolérance des allogreffes hépatiques dans certaines espèces animales ne se retrouvent chez l'homme que sous forme de nuances. Ces nuances, loin d'être négligeables, font de l'allogreffe hépatique l'allogreffe d'organe la mieux tolérée mais ne font pas différer fondamentalement l'attitude thérapeutique vis-à-vis du malade ayant subi une transplantation hépatique de celle que l'on peut avoir visà-vis de ceux ayant eu une transplantation rénale ou cardiaque: il est nécessaire d'utiliser un traitement immunosuppresseur pour prévenir les crises de rejet aigu qui sont fréquentes.

Plusieurs facteurs allant dans le sens d'une meilleure tolérance des allogreffes hépatiques chez l'homme (et qui sont ainsi éclairés par les données de l'expérimentation animale) doivent cependant être soulignés. Pour des raisons pratiques qui tiennent surtout à la durée maximale d'ischémie froide tolérable par le greffon hépatique, qui est actuellement d'environ six heures, il n'est pas possible d'effectuer à temps les tests immunologiques (typage HLA $^{*}$, recherche chez le receveur d'anticorps cytotoxiques contre le donneur) qui, en transplantation rénale, permettent d'apparier au mieux donneur et receveur. Pour un donneur précis, la sélection du meil-

* Human leucocyte antigen. 


\section{RÉFÉRENCES}

14. Tsurufuji, Ishigara $\mathrm{K}$, Shinọmiya $\mathrm{T}$, Uchida T, Kamada N. Immunosupressive activity of serum from liver grafted rats: in vitro specific inhibition of mixed lymphocyte reactivity by antibodies against class II RTl alloantigens. Immunology 1987 ; 61 : 421-8.

15. Fabre JW, Milton AD, Spencer SC, Settaf A, Houssin D. Regulation of alloantigen expression in different tissues. Transplant Proc 1987 ; 19 : 200-6.

16. Settaf A, Milton AD, Spencer SC, Houssin D, Fabre JW. Donor class I and class II MHC antigen expression following liver allografting in rejecting and non rejecting strain combinations Transplantation 1988 (sous presse).

17. Gassel HJ, Engemann R, Hutchinson IV, Morris PJ. Successful retransplantation of arterialized rat liver grafts. Eur Surg Res $1987 ; 46: 132$

18. Houssin D, Charpentier B, Lang P, et al. In vivo and in vitro correlates of the specific transplantation tolerance induced by spontaneously tolerated liver allografts in inbred strains of rats. Transplant Proc 1981 ; 13 : 61922.

19. Kamada N, Davies HffS, Roser B : Reversal of transplantation immunity by liver grafting. Nature $1981 ; 292: 840-2$.

20. Houssin D, Bellon B, Brunaud MD, et al. Interactions between liver allograft and lymphocytotoxic antibodies: an experimental study in the rat. Hepatology $1986 ; 6: 994-8$.

21. Settaf A., Meriggi F, Van de Stadt J, et al. Delayed rejection of liver xenografts compared to heart xenografts in the rat. Transplant Proc 1987; 19 : 1155-8.

22. Iwatsuki S, Iwaki Y, Kano T et al. : Successful liver transplantation from cross-match positive donors. Transplant Proc 1981; 13 : $281-5$

23. Gordon RD, Iwatsuki S, Esquivel CO, Bokis A, Todo S, Starzl TE. Liver transplantation across ABO blood groups. Surgery $1986 ; 100: 342-8$.

24. Starzl TE. Experience in hepatic transplantation. Philadelphia: WB Saunders, 1969: 415 .

25. Mohanakumar T, Rhodes C, Mendez-Pi con G, Flye MW, Lee HM. Antiidiotypic antibodies to human major histocompatibility complex class I and II antibodies in hepatic transplantation and their role in allograft

leur receveur possible du point de vue immunologique n'est donc pas faisable actuellement. Des études rétrospectives ont montré qu'à court ou moyen terme, les résultats n'apparaissaient pas significativement différents, que la compatibilité dans le système HLA soit bonne ou mauvaise, et surtout qu'il y ait eu ou non chez le receveur présence d'anticorps lymphocytotoxiques dirigés contre les antigènes du donneur [22].

Pour ces raisons, le seul mode de sélection du receveur en fonction du donneur qui soit actuellement utilisé en vue d'une transplantation hépatique, est la recherche d'une compatibilité dans le système des groupes sanguins $\mathrm{ABO}$. Un aspect spectaculaire de la tolérance des allogreffes de foie chez l'homme est que, lorsque pour des raisons d'urgence, des malades ont reçu un greffon hépatique provenant d'un donneur $\mathrm{ABO}$ incompatible, les résultats immédiats et à distance n'ont pas été significativement différents de ceux observés chez les malades ayant reçu un greffon compatible [23]. Quoique les données soient très limitées, il est possible que la résistance du foie aux anticorps cytotoxiques, dont nous avons évoqué les aspects chez l'animal et chez l'homme, se traduise également dans le domaine de la transplantation xénogénique chez l'homme. En 1966, Starzl et al. [24] avaient, dans une situation d'extrême urgence, mis en place le foie d'un chimpanzé chez un enfant de deux ans. Chez ce malade dans un état très grave et soumis à un intense traitement immunosuppresseur, l'évolution post-opératoire fut marquée par des complications infectieuses qui entraînèrent le décès en neuf jours : rien ne permet cependant d'affirmer que ce greffon xénogénique ait été la cible d'un rejet particulièrement important.

Dans l'ensemble, le traitement immunosuppresseur utilisé après transplantation hépatique s'inspire de celui utilisé en transplantation rénale ou cardiaque. Cependant, les doses de départ comme d'entretien sont souvent plus faibles, les échecs liés à un rejet aigu incontrôlable ou à un rejet chronique sont peu fréquents. Ce traitement immunosuppresseur rend difficile la détection d'un état de tolérance analogue à ce qui a été observé chez l'animal. D'autre part, l'expérience clinique en matière de transplantation simultanée chez un receveur de foie d'un autre organe (rein, cœur ou bloc cœur-poumon) provenant du même donneur est trop limitée et débutante pour qu'un éventuel effet protecteur lié à l'allogreffe de foie ait pu être démontré. Différents mécanismes ont été évoqués pour expliquer la situation favorable du greffon hépatique face au rejet chez l'homme, parmi lesquels nous pouvons citer la régulation anti-idiotypique de la réponse immune aux antigènes du $\mathrm{CMH}$ [25], et l'adaptation du greffon par remplacement des cellules de Kupffer du donneur par celles de l'hôte.

Malheureusement la tolérance des allogreffes hépatiques pourrait aussi avoir des aspects néfastes. Chez les malades ayant reçu une greffe de foie hépatique pour une affection hépatique tumorale maligne, le pourcentage de malades faisant une récidive de leur maladie néoplasique au niveau du greffon est très élevé. Existe-t-il un facteur d'environnement local qui, tout en favorisant l'acceptation du greffon, pourrait également favoriser l'implantation à son niveau des cellules néoplasiques?

\section{Conclusion}

La tolérance des greffes hépatiques expérimentales est un phénomène immunologique très surprenant et passionnant. Reproduire le mécanisme qui induit cette tolérance est une idée séduisante pour celui qui rêve, pour les malades, d'un état de tolérance de transplantation spécifique des antigènes du donneur qui rendrait superflu le traitement immunosuppresseur. La tolérance des greffes hépatiques chez l'homme est actuellement une consolation qui vient compenser la complexité de l'acte chirurgical et son retentissement. C'est aussi le souhait que lorsque la compatibilité entre donneur et receveur pourra être la plus ajustée possible, les résultats de la transplantation seront encore meilleurs. C'est enfin l'espoir qu'un projet aussi peu raisonnable que la transplantation hépatique xénogénique devienne un jour réalité 


\section{Summary}

Liver grafts are less sensitive to rejection than kidney, heart or pancreatic grafts. Tolerance of liver grafts has several aspects. Experimentally, in pigs and particularly in rats, liver allografts may become spontaneously tolerated and in rats may induce a donor specific state of transplantation tolerance. Liver grafts appear particularly resistant to lymphocytotoxic allogenic antibodies and also to xenogenic natural antibodies. In man, tolerance to liver allograft is less spectacular than in rat; however it is illustrated by the observation that liver allografts can be done without damage in case of a positive cross match or in case of an $\mathrm{ABO}$ incompatibility between donor and recipient.

\section{Constitution d'un fichier de volontaires au don de moelle osseuse}

Les greffes réalisées en France ont jusqu'à présent été faites à partir d'un donneur familial. En 1987 environ 500 greffes de moelle osseuse auront été effectuées. Des progrès récents permettent d'envisager la transfusion de cellules médullaires prélevées chez des donneurs non apparentés.

En effet, seuls $30 \%$ des patients devant bénéficier d'une greffe, auront dans leur fratrie un donneur HLA identique. Dans certaines indications et en particulier les leucémies myéloïdes chroniques, les aplasies, les déficits combinés sévères, des greffes à partir de donneur non apparenté ont été réalisées [1, 2]. Les premiers résultats étant encourageants [3], il est apparu nécessaire de constituer en France un fichier de volontaires au don de moelle osseuse comme il en existe dans d'autres pays notamment aux États-Unis [4-6].

Depuis environ deux ans, différents moyens ont été utilisés pour informer l'ensemble de la population française et non pas une certaine catégorie de donneurs potentiels qu'auraient pu être les donneurs de sang. Ces moyens ont transités par l'intermédiaire de bénévoles de la FFDOT (fédération française des donneurs d'organes et de tissus humains) et des pharmaciens ayant accepté de distribuer des formulaires d'information.

D'autres structures d'information se sont progressivement ajoutées telles que services d'accueil des centres de transfusion sanguine et associations de malades.
Chaque information est faite à deux niveaux : tout d'abord l'éventuel volontaire demande des renseignements qui lui permettront de connaître les réponses à la plupart des questions qu'il peut se poser: qu'est-ce que la moelle osseuse, quelles sont les contraintes d'un don anonyme et bénévole, etc., ensuite à sa demande il reçoit un formulaire comportant un consentement sur lequel le donneur s'engage à devenir volontaire jusqu'à l'âge de 50 ans pour un malade qu'il ne connaîtra jamais; ce consentement comporte par ailleurs des informations concernant le risque anesthésique et les délais de convocation dans le laboratoire devant réaliser les examens d'histocompatibilité. Seul le typage HLA$A$ et $B$ est fait pour entrer dans le fichier.

A ce jour, plus de 30000 demandes de renseignements ont été adressées par l'intermédiaire de la FFDOT. Les associations de malades, très actives, ont permis par ailleurs dans certaines régions le recueil d'un grand nombre de consentements. Un écueil pourtant est apparu très vite, c'est l'inadéquation entre le désir des familles de malades de constituer très rapidement ce fichier et la "nécessaire» lenteur pour la réalisation des groupes HLA. La seconde difficulté concerne les volontaires recrutés par des médias ne décrivant pas réellement les contraintes d'un tel don, c'est-à-dire don pour un malade anonyme: beaucoup veulent donner à un malade en 